\title{
Blood Purification for Severe Hepatitis in Vivo and Vitro
}

\author{
Ming Luo, Xian-jun Zhang and Wu-kui Cao
}

Exterior and interior blood purification (EIBP) refers to the combined therapy of interior blood purification (IBP) and exterior blood purification (EBP) for severe hepatitis. For one thing, integrated traditional Chinese and Western medicine therapy should be applied in IBP to reduce and prevent the generation and absorption of harmful substances in vivo. For another, EBP such as the artificial liver support system (ALSS) can extract blood from the body and various kinds of existing harmful substances in the blood can be cleared in vitro, using the treatment of plasma exchange, plasma perfusion, plasma adsorption, hemofiltration, hemodialysis. Therefore, IBP should be cooperated with EBP for the treatment of severe hepatitis, and EIBP can prevent the deterioration of this disease and lower the mortality of patients.

Key words: Severe hepatitis; Blood purification; Vivo; Vitro

$\mathrm{T}$ The theoretical system of exterior and interior blood purification (EIBP) was proposed by Prof. Wu-kui Cao from Tianjin Infectious Diseases Hospital in 2005. EIBP refers to a therapeutic method of reducing and preventing intestinal endotoxin from entering the blood, which can clear various kinds of harmful substances existing in the blood. ${ }^{1}$ On one hand, integrated traditional Chinese and Western medicine therapy should be applied in the interior blood purification (IBP). For example, antibiotics can inhibit the growth of bacteria that produce endotoxin, and traditional Chinese agents are able to promote intestine peristalsis to reduce the absorption of endotoxin. In addition, colonic enema and dialysis are applied to clear the intestinal tract, while microecological agents are capable of regulating and reconstructing intestinal flora. According to the source, generation pathways and absorption links of harmful substances, IBP should be performed to reduce and prevent the generation and absorption of the substances leading to purify the blood. On the other hand, power devices such as the artificial liver support system (ALSS) can extract blood from the body and various kinds of existing harmful substances such as endotoxin in the blood can be cleared through the treatment of plasma exchange, plasma perfusion, plasma adsorption, hemofiltration, hemodialysis and bio-ALSS. These therapeutic methods of severe hepatitis are considered as the exterior blood purification (EBP), and the combined therapy of IBP and EBP for severe hepatitis is called EIBP.

\section{BACKGROUND OF EIBP}

A growing number of clinical studies indicated that the application of EBP such as plasma exchange, has obtained certain therapeutic effect in the treatment of severe hepatitis. Through the removal of the endotoxin, immune complex, lipid peroxide, tumor necrosis factors and bilirubin in the blood, EBP can alleviate the hepatocellular damage caused by these harmful substances. At the same time, fresh plasma and albumin are infused for complement of coagulation factors, opsonin and nutrients promoting the restoration and regeneration of hepatocytes. Especially, the invention and application of bio-ALSS with biological synthesis and transformation functions has further improved the therapeutic effect for severe hepatitis. However, it is found that EBP cannot reduce and prevent the continuous generation and absorption of gut-derived harmful substances such as endotoxin, ammonia and various kinds of inflammatory factors stimulated by the substances. Due to the sustained damage of liver and other organs, the severity of severe hepatitis is likely to deteriorate further. ${ }^{1}$ Therefore, the theory of EIBP is proposed by the authors in order to make up the disadvantages of EBP. According to the source, generation pathways and absorption links of harmful substances, IBP is performed with EBP to reduce and prevent the continuous generation and absorption of the substances, which can alleviate the sustained liver damage and reduce the mortality of severe hepatitis.

Tianjin Liver Disease Institute, Tianjin Infectious Disease Hospital, 


\section{THEORETICAL BASIS OF EIBP FOR SEVERE HEPATITIS}

Severe hepatitis can be caused by a variety of etiologies. Acute or chronic hepatotropic virus infection, alcoholism, administration of drugs with hepatic toxicity, metabolic diseases, autoimmune diseases, liver tumor, surgery and serious infection can cause and aggravate liver damage leading to liver failure in severe cases. Hepatitis viruses are the most etiology of severe hepatitis in China, especially hepatitis B virus (about $70 \%$ ). Generally speaking, different kinds of harmful substances including gut-derived endotoxin exist in the blood of patients with severe hepatitis. For healthy adults, the mechanical, biological and immune barriers of the intestinal mucosa can reduce the absorption of endotoxin, which prevents the occurrence of intestinal endotoxemia. However, these barriers are damaged and the mucosal permeability increases significantly in patients with severe hepatitis. ${ }^{2}$ Therefore, bacteria and endotoxin can translocate from the intestinal tract through damaged mucosa and enter the systemic circulation by the portal vein and the mesenteric lymph system. Though hepatic Kupffer cells have the ability of clearing endotoxin, the cells are also stimulated by endotoxin and then produce a large amount of cytokines and inflammatory mediators that give the "second strike" to liver through many kinds of pathways. Besides, the theory of intestinal microecology is also the theoretical basis of IBP. ${ }^{5}$ Liver function and intestinal microecology system can cooperate with each other. Normal liver function is the guarantee of maintaining intestinal flora balanced, and many studies found intestinal microecological disorder is closely related to severe hepatitis. The number of obligate anaerobic bacteria such as bifidobacterium and bacteroides decreases significantly, but the amount of gram-positive bacteria markedly increases in patients with severe hepatitis, which leads to the increase of endotoxin. Excessive endotoxin translocates through the damaged intestinal mucosa and finally results in intestinal endotoxemia. Thus, a vicious circle is made up of liver diseases, intestinal microecological disorder and intestinal endotoxin, which plays a key role in the occurrence and deterioration of severe hepatitis. The ALSS only clears the harmful substances already existing in the blood, but it can not reduce and prevent continuous generation and absorption of intestinal endotoxin and inflammatory factors that can cause sustained damage of liver and other organs. Therefore, ALSS should be combined with IBP to make up the disadvantages in the treatment of severe hepatitis.

\section{IBP TREATMENT FOR SEVERE HEPATITIS}

IBP can inhibit the overgrowth of intestinal bacteria, prevent the generation and absorption of endotoxin, promote intestinal emptying, decompose the absorbed endotoxin and enhance the function of reticuloendothelial system.

\section{Western medicine treatment}

Antibiotics, biological products and combined therapy

Shi et al suggested that the oral narrow-spectrum antibiotics could be taken to clear gram-negative bacteria and fungus in the intestinal tract, protect the obligate anaerobic bacteria, reduce the antibiotic resistance of facultative anaerobic gram-negative bacteria and enhance the barrier function of intestinal mucosa, which conduce to inhibit bacterial translocation and lower the occurrence rate of infection and endotoxemia. ${ }^{6}$ Compared with the treatment only using antibiotics, the combined therapy of IBP and biological products such as lipid A antagonists, LPS monoclonal antibodies and anti-endotoxin antibodies can significantly lower the levels of endotoxin and cytokines in the blood. It provides a new therapeutic method for endotoxemia and its complications. ${ }^{7}$

\section{Gastric prokinetic medicine}

For example, cisapride can promote the release of acetylcholine from the intestinal myenteric plexus and improve the intestinal motility and bile secretion. Therefore, this kind of medicine has a certain therapeutic effect for intestinal endotoxemia.

\section{Amino acids}

Glutamine provides $70 \%$ energy for the proliferation of intestinal epithelial cells, and the its supplement can inhibit the apoptosis induced by oxygen-free radicals. Glycine prevents the degranulation of intestinal mast cells, reduces the release of histamine and lowers the permeability of intestinal wall, which may help reduce the absorption of intestinal endotoxin. ${ }^{2}$

\section{Epidermal growth factor (EGF)}

The biological activity of EGF is implemented through combination with specific receptors. EGF can promote the proliferation of intestinal epithelial cells and increase the transport and absorption of nutrients and electrolytes in these cells.

\section{Traditional Chinese medicine (TCM) treatment}

TCM includes single and compound medicine. Studies of single medicine suggest that Rhubarb, Dandelion, Dandelion, Radix scutella riae, Isatis root and Radix scrophulariae have direct or indirect anti-endotoxin 
effect in vitro. Astragalus, Ginseng and Ligusticum chuanxiong can promote endotoxin clearing, while Radix scutella riae, Dandelion, Isatis root and Sophora tonkinensis can decompose endotoxin. Furthermore, Rhubarb, Honeysuckle and Astragalus can inhibit the overgrowth of intestinal Escherichia coli and reduce the generation and absorption of endotoxin. Studies on compound medicine showed that a compound traditional Chinese agent named Shuangligan can significantly decrease the levels of plasma endotoxin in rats with liver injury, and other agents called $\mathrm{Da}$ chengqi decoction is capable of blocking the biological effects of intestinal endotoxemia in rats with acute liver injury. In addition, a prescription of TCM named Cooling blood to dissipate blood stasis can inhibit the hepatocellular apoptosis induced by endotoxin in liver injury.

\section{Intestinal cleaning}

\section{Retention enema by western medicine}

Western medicine can mainly decrease intestinal $\mathrm{pH}$ values, enhance catharsis and regulate intestinal microecology. The commonly used medicine are lactulose, acetic acid, antibiotics, magnesium sulfate and intestinal microecological agents.

\section{Retention enema by TCM}

Based on the theory of syndrome differentiation in TCM, there are many studies on the prescriptions of TCM for enema, which showed significant therapeutic effects for severe hepatitis. TCM can promote the intestinal peristalsis, enhance the excretion of ammonia and reduce the generation and absorption of intestinal endotoxin resulting in purifying the blood.

\section{Colonic lavement}

Colonic therapy system is applied to perform the high colonic lavement in which the lavage probe is implanted into the colonic cavity for more than 50 centimeters. Colonic lavement can alleviate the symptoms of intestinal endotoxemia, inhibit the generation of ammonia and clear metabolic products by the characteristics of semi-permeable colon mucosa.

\section{Colonic dialysis}

Colonic mucosa is considered as the natural semipermeable membrane, and the dialysate exchanges the harmful substances with blood in the lower layer of colon mucosa. High-concentration toxin moleculers in blood are exchanged into the colonic cavity and then discharged from the body. Moreover, TCM can be added in the dialysate and spread to blood resulting the therapeutic effect.

\section{Regulation of intestinal flora}

Microecological therapy is a positive and effective method for intestinal tract clearance in patients with severe hepatitis. Hepatic dysfunction and intestinal microecological disorder contact and influence each other, with cause-and-effect relationship between them. Therefore, intestinal microecological agents play a positive role in liver protection. In the treatment of severe hepatitis, these agents can regulate the intestinal microecological disorder to keep the microecology balanced and stable, which reduces the endotoxin absorption and ammonia. Commonly used agents are bifidobacterium, lactobacillus, enterococcus and enterococcus faecalis, which can lower intestinal $\mathrm{pH}$ values and inhabit the colonization and overgrowth of gram-negative bacteria reducing the generation and absorption of endotoxin. In addition, synbiotics are made up of triple viable bacteria (probiotics) and growth-enhancing factors (prebiotics), which can exert the physiological activity of probiotics and selectively promote the growth of probiotics. For patients with severe hepatitis and minimal hepatic encephalopathy, symbiotics are able to improve liver functions and prevent the occurrence of hepatic encephalopathy. Microecological agents are one of the hot issues in clinical practices, but there is no ideal agent for the treatment of severe hepatitis. Many new agents are still in experimental stages, and the mechanisms of their therapeutic effects have not been clarified yet. According to the development and pathophysiological characteristics of severe hepatitis, ideal microecological agents must ensure the administration safety without poisonous and side-effects in long-term treatment, and they ought to have the blocking effect on the pathogenic mechanisms of endotoxin.

\section{COMBINED THERAPY OF IBP AND EBP}

Severe hepatitis should be treated through combined therapy of IBP and EBP. According to the source, generation pathways and absorption links of harmful substances, IBP can reduce and prevent continuous generation and absorption of the substances. At the same time, EBP is able to directly clear the existing endotoxin, bilirubin, inflammatory factors and metabolites in the blood. The therapeutic effect of combined therapy is significantly superior to that of single method.

Many scholars have combined the application of ALSS with the oral administration, colonic lavement and enema of western and traditional Chinese agents in the treatment of severe hepatitis, which showed significantly therapeutic effects. ${ }^{8,9}$ Huang et al reported that patients with severe hepatitis were treated by nonbio-ALSS, intravenous injection of Yinzhihuang and retention enema by rhubarb, which significantly reduced the absorption of endogenous endotoxin and inhibited the occurrence of inflammatory response syndrome. ${ }^{10}$ Based on the internal comprehensive 
treatment, Zhao et al reported that combined therapy of nonbio-ALSS and virgate wormwood decoction in the treatment of severe hepatitis, can achieve the therapeutic response rate of $88.89 \%{ }^{11} \mathrm{Li}$ et al reported that patients with severe hepatitis and endotoxemia were treated by combined therapy of colonic lavage and plasma exchange, and the results suggested that the declined level of serum endotoxin and blood ammonia, the improvement of liver function and the survival rate in the combined therapy group were significantly higher than that of the plasma exchange group and the control group. ${ }^{12}$ Furthermore, ALSS was applied with fresh rehmannia juice in treatment of severe hepatitis in the study by Li et al, and the result showed the levels of serum bilirubin, endotoxin and cytokines significantly decreased in the combined therapy group $(P<0.05$ or $P<0.01){ }^{13}$ Others reported that 32 patients with severe hepatitis were treated by EBP and TCM, which proved that the therapeutic effect of EIBP was superior to that of EBP. Especially in the early stage of severe hepatitis, the application of IBP can effectively decrease the concentration of harmful substances and improve the clinical symptoms and lower the mortality. ${ }^{1}$

In conclusion, the authors have proposed the comprehensive treatment system for severe hepatitis. Based on the ALSS, TCM and microecological agents can regulate intestinal flora and reduce the absorption of endotoxin, which can purify the blood in vivo and vitro. Western and traditional Chinese agents can be directly absorbed through the intestinal administration, and can reduce the generation and absorption of intestinal endotoxin and avoid side-effects such as vomiting, diarrhea and liver overload. Some agents can promote intestinal peristalsis, lower the intestinal $\mathrm{pH}$ values, inhibit the overgrowth of bacteria and maintain the microecological balance in the intestinal tract. Meanwhile, other agents are able to improve the blood perfusion of the gastrointestinal mucosa, promote the repairment of intestinal mucosa injury, inhibit the injury caused by cytokines and inflammation mediators and prevent the translocation of bacteria and the occurrence of endotoxemia. Moreover, the application of colonic irrigation and colonic dialysis can clear harmful substances that are not detoxified by liver. With the characteristics of semi-permeable colonic mucosa, high-concentrations toxin molecules are exchanged into colonic cavity and then discharged from the body. These methods can alleviate the symptoms of intestinal endotoxemia, reduce enterohepatic circulation of bilirubin and inhibit the generation of ammonia, which help purify the blood in vivo. According to the source, generation pathways and absorption links of harmful substance, IBP should be cooperated with EBP for the treatment of severe hepatitis. EIBP can prevent the progression of severe hepatitis in the suspected patients and lower the mortality of patients in early, interim and later stages of this disease.

\section{REFERENCES}

1. Cao WK, Yuan GY, Tang KC, Li Q. Clinical study on blood purification in severe hepatitis in vivo and vitro. $\mathrm{J}$ Tianjin Med 2005;33:642-643.

2. Liu XQ. Importance of intestinal barrier dysfunction in the deterioration of liver cirrhosis. J Heilongjiang Med 2008;21:41-43.

3. Ai T, Tian DY. Severe hepatitis and intestinal endotoxemia. J Internal Intens Med 2007;13:322-324.

4. Li J. The second hit of endotoxin to liver and traditional Chinese medicine treatment. J Beijing Tradit Chin Med 2008;27:94-95.

5. Li LJ, Wu ZW, Ma WH, Yu YS, Chen YG. Study on the changes of the intestinal flora in patients with chronic severe hepatitis. J Chin Infect Dis 2001;19:345-347.

6. Chen HS, Liu WJ. The research progress of anti-endotoxin drugs. World Notes Antibio 2002;23:174-177.

7. Shi WX, Yang JZ, Yang SM. Study on the anti-endotoxin agents and the induction of endotoxins by antibacterials. J Chin Antibio 2007;32:454-459.

8. He JC, Shao Y, Zhang B, Shao ZC. Research progress on the treatment of endotoxemia. J Med Res 2008;37:102-104.

9. Ji AY, Ma SP, Zhao WX, Yang GH, Zhang ZL, Zhang YY, et al. The ALSS combined with traditional Chinese and Western medicine in the treatment of 63 patients with severe hepatitis. J Chin Integrat Tradit West Med in Intens Critic Care 2005;12:314-315.

10. Huang XJ, Yang ZH, Huang XJ, Li XY. The therapeutic method of non-biologic artifical liver support system combined with traditional Chinese medicine for severe hepatitis. Tradit Chin Medi Res 2007;20:2-5.

11. Zhao Y, Liu FQ, Zhang HT, Zhang XY, Liu WJ. The therapeutic effects of the non-biologic blood purification technology combined with yinchenhao decoction in 162 patients with severe hepatitis.Chin J Dialys Artif Organs 2006;17:40-41.

12. Li WY, Zhang ZG, Qian YD, Tian DY. Clinical study of the coloclyster combined with plasma exchange in patients of severe chronic hepatitis. Chin J Integrat Tradit West Med Liver Dis 2008;18:25-26.

13. Li Q, Yuan GY, Jia JW, Tang KC, Zhao J. Research of the therapeutic effect of the ALSS combined with fresh rehmannia juice in the treatment of severe hepatitis. Chin J Dialys Artif Organs 2007;18:1-4. 